\title{
Personalizing therapy for colorectal cancer
}
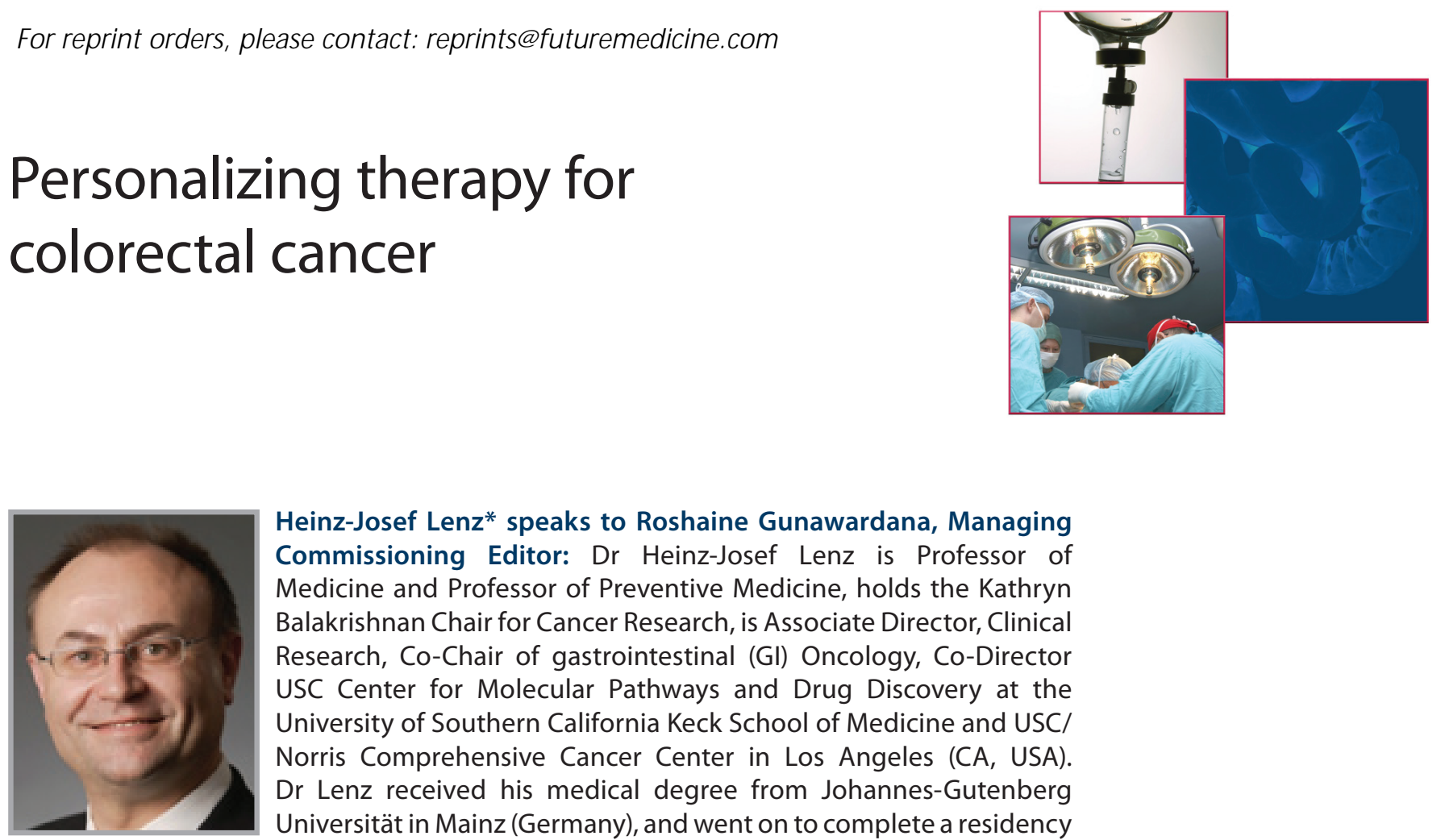

Heinz-Josef Lenz* speaks to Roshaine Gunawardana, Managing Commissioning Editor: Dr Heinz-Josef Lenz is Professor of Medicine and Professor of Preventive Medicine, holds the Kathryn Balakrishnan Chair for Cancer Research, is Associate Director, Clinical Research, Co-Chair of gastrointestinal (GI) Oncology, Co-Director USC Center for Molecular Pathways and Drug Discovery at the University of Southern California Keck School of Medicine and USC/ Norris Comprehensive Cancer Center in Los Angeles (CA, USA). Dr Lenz received his medical degree from Johannes-Gutenberg Universität in Mainz (Germany), and went on to complete a residency in hematology and oncology at the Eberhard-Karls Universität Tübingen (Germany), before completing clerkships in oncology and hematology at George Washington University (DC, USA) and the Beth Israel Hospital of Harvard Medical School (MA, USA), respectively. He completed a postdoctoral Fellowship in the Department of Biochemistry and Molecular Biology at the USC Keck School of Medicine. Dr Lenz's research focus is in the identification of predictive and prognostic biomarkers in GI cancers, early drug development and novel clinical trials. He is co-chair of SWOG GI Committee, Chair SWOG GI Translational Medicine and member of the National Cancer Institute ( $\mathrm{NCl})$ Gl Steering Committee, $\mathrm{NCl}$ Investigational Drug Steering Committee, the $\mathrm{NCl}$ Gastroesophageal Task Force and $\mathrm{NCl}$ Correlative Science Committee. He is the Principal Investigator for R01, U01, U10 and USC PI for N01. He has been awarded a number of prestigious awards including American Society of Clinical Oncology (ASCO) Young Investigator Award, ASCO Career Development Award, NCl Career Development Award, STOP Cancer Career Development Award and Research Recognition Award. He has published over 270 peer reviewed papers including Nature Medicine, Nature Genetics, Journal of Clinical Oncology and Lancet.

Q The last time we interviewed you, we spoke of how your group in particular is interested in differences seen in specific ethnic populations, in relation to predicting outcomes. How is this research progressing? We are making very good progress and have identified significant differences in the activation of key pathways in angiogenesis between Hispanics, Caucasians and Japanese which may explain in part the differences in outcome using anti VEGF therapies. We are working with investigators of FIRE3, TRIBE, MAVERICC and 80,405 to complete genome-wide association studies (GWAS) analyses on almost 2000 patients which further help us to understand the value of germline alterations in the outcome of patients with metastatic disease. We have identified novel single nucleotide polymorphisms prediciting toxicity to anti-EGFR and anti-VEGF which appear to have differences in frequencies in different ethnic populations. We hope to present some of these data at American Society of Clinical Oncology or European Society for Medical Oncology next year.

*Norris Comprehensive Cancer Center, Keck School of Medicine, 1441 Eastlake Ave, Suite 3456, University of Southern California, Los Angeles, CA 90089, USA; lenz@hsc.usc.edu 
Q In today's environment, how important do you think it is to be able to further define at-risk patient populations \& develop personalized chemopreventive strategies? There have been significant efforts to identify the risk population for colon cancer and we have learned a lot and refined the way we are screening for genetic predisposition and enroll these high risk patients into highly effective screening protocols. However, we have not developed very effectively chemoprevention strategies beyond celecoxib or sulindac. For the general population with some increased risk but no genetic predisposition we are struggling to find easy prevention interventions. We know exercise, reduced alcohol consumption and decreased red meat are critical. Recent data showed that coffee consumption decreases colon cancer risk, I guess everything which makes your bowel move is decreasing risk. What we really need is individual prevention strategies based on the individual risk profile.

Q Do you find that today's cancer patient is more well informed when they come to the clinic, or do you think that with the abundance of information now more freely available, that they can sometimes be misinformed? How do you think this is affecting doctor-patient relationships?

There is so much information available for patients and doctors which can be very easily confusing particular when it is filled with anecdotal examples of cure with out-of-standard therapies. One of the challenging problems is how to read the information available and how to sort them into information you can rely on and build on. I experience that many of my patients are lost in the different information available and particular was being understood about their prognosis. When patients look up stage 4 disease, they are all depressed not understanding what these numbers really mean and how we utilize these numbers for treatment decisions. What I find very difficult is that many family members and friends find in the internet all these supplements and alternative therapies which promise to cure cancer and provide this information to the patient who is overwhelmed and does not know what data to trust. Here, the physician plays a critical role to make recommendations and guide the patient and family through this jungle of confusing information. The better informed the patient the better treatment options are understood, and discussions are much more effective.

\section{Q One of your recent papers was on} integrin- $\beta$-3 genetic variants $\&$ risk of venous thromboembolism in colorectal cancer patients. Can you briefly describe your findings to our readers?

We have been very interested to better understand specific side effects of the new targeted agents such as bevacizumab and cetuximab. We have studied almost 1000 patients undergoing first line therapy. Very few of these patients may experience life threatening toxicity such as gastrointestinal perforation, gastrointestinal bleeding, arterial thromboembolic events and others. We were able to identify germline variations in genes which can predict these side effects such as the intergrin single nucleotide polymorphism predicting thromboembolism. We are in the middle of validation of these data in an independent cohort. Interestingly we found that patients who developed these side effects did live significantly longer.

\section{Q There have been a lot of research carried} out recently on circulating tumor cells \& circulating DNA. What is your opinion on the prognostic value of circulating tumor cells \& how does the research into this need to evolve over the next few years?

Liquid biopsies will revolutionize the way we diagnose and monitor our patients in the future. We are now able to collect $10 \mathrm{cc}$ blood and detect not only circulating tumor cells, but also cell free DNA and cell-free RNA to identify molecular mechanisms of response/resistance. New technologies are now able to molecular classify single cells with the goal to understand what cells survive our chemotherapy and their molecular make up to design novel therapeutic strategies. We can with liquid biopsies (simple blood draw) monitor in real time changes under therapy to identify early when therapy does not work and how tumor cells can overcome this therapy informing us what the most effective subsequent therapies could be. There is no doubt in my mind this will be used routinely in our practices in the future.

\section{Q How can we make genomic testing for colorectal cancer more cost effective?}

Volume. The more patients we test the more automated the systems will become and the lower the cost. However, the question should be another one how cost effective is it to use 
genomic testing by using a more effective therapies avoiding toxic regimens, these test even not cheap may save us significantly overall but avoiding costly therapies. It will also speed up drug development which is a main part of the costs for getting drugs approved.

\section{Q What is the future of TAS-102 in your opinion based on the studies carried out so far?}

In colon cancer we have been fortunate that we have access to novel therapies. A couple of years ago with regorafenib and since October also TAS102. This is another oral anticancer drug which has shown in a recent Phase III study significant efficacy in increasing progression-free survival and overall survival for patients who had all standard of care therapies. TAS102 will become part of our armentarium in the treatment of metastatic colorectal cancer and many new trials are being initiated to test TAS102 in combination with other cytotoxic or targeted agents to further increase benefit from this novel cytotoxic compound.

Q Do you have any concluding remarks for our readers regarding personalized therapeutic approaches \& outcomes prediction for colorectal cancer?

We are experiencing a molecular revolution in cancer research and in colorectal cancer.
Having the data from the Cancer Genome Atlas is only the beginning of the molecular characterization of this very heterogeneous disease. New technologies doing whole genome sequencing or single cell molecular characterization or liquid biopsies will be the basis for a new generation of clinical trials taking advantage of a better selection of patients who benefit the most of specific drug targeting activated pathways or mutations. It will take time to integrate these technologies and validate molecular signatures to be used in our clinical practice but we are on our way. It is a very exciting time to witness the molecular transformation of our pathological staging and prognostication translating into new therapies.

\section{Disclaimer}

The opinions expressed in this article are those of the author and do not necessarily reflect the views of Future Medicine Ltd.

Financial \& competing interests disclosure

H-J Lenz has no relevant affliations or financial involvement with any organization or entity with a financial interest in or financial conflict with the subject matter or materials discussed in the manuscript. This includes employment, consultancies, honoraria, stock ownership or options, expert testimony, grants or patents received or pending, or royalties.

No writing assistance was utilized in the production of this manuscript. 